\title{
UNIDIRECTIONAL INFILTRATION \\ IN DEFORMABLE POROUS MEDIA: MATHEMATICAL MODELING AND SELF-SIMILAR SOLUTION
}

\author{
$\mathrm{BY}$ \\ L. BILLI \\ AND
}

A. FARINA (Dipartimento Ingegneria Aeronautica e Spaziale, Politecnico di Torino, Italy)

\begin{abstract}
This study is motivated by the necessity to develop models and to obtain mathematical results which can help in improving certain industrial processes used for manufacturing composite materials. We focus on unidirectional infiltration processes in deformable porous material and on the analytical study of the related mathematical problem. A key point in developing the model is the use of a set of Lagrangian coordinates fixed on the solid. This technique allows us to simplify the mathematical problem. In the Eulerian formalism, in fact, such a problem is a free boundary problem characterized by the presence of two time-dependent interfaces. The use of material coordinates fixed on the solid allows, vice-versa, to fix one boundary and so to obtain a nonlinear one-phase Stefan problem. The latter, then, has been studied from the analytical viewpoint. In particular, we have proved the existence of a solution exhibiting a self-similar form.
\end{abstract}

1. Introduction. In the present paper we focus on a certain class of industrial processes used to manufacture composite materials. The aims of the work are to develop a mathematical model for this kind of process and to study, from the analytical viewpoint, the related mathematical problem.

Several composite materials are manufactured using some industrial processes, usually named Resin Transfer Molding (RTM), Structural Resin Injection Molding (SRIM), or Squeeze Casting (SC), which essentially consists of injecting a metallic, ceramic, or polymeric melt in a porous preform of reinforcing elements [1], [2]. After the liquid matrix fills up the preform, it solidifies holding the reinforcing elements together and enabling the transfer of the major stresses and loads to the solid preform.

Received February 17, 1998.

2000 Mathematics Subject Classification. Primary 76S05.

The first author wrote the article while he was visiting OCIAM Mathematical Institute, Oxford, UK, thanks to a grant of the University of Rome "La Sapienza". 
It appears from the literature (see, for example, [3], [4]) that the identification of the production cycle to be used for manufacturing composites by means of the above techniques is still more an art than a science and has to be developed every time on a trial-and-error basis. It is therefore fundamental, in order to improve these manufacturing procedures, to develop models and to study the correct formulation of the related mathematical problems. This paper is therefore devoted to settling a model for the above-mentioned processes and to analyzing the mathematical problem generated by the model itself.

The starting point in building the model, as suggested by the physical phenomena involved in RTM, SRIM, or SC, is the theory of flows in deformable porous media. When a fluid flows through a deformable porous medium, in the present case the solid preform, the forces associated with the flow deform the porous material. In turn, the deformation of the porous medium influences the flow. The competition among the stress in the solid element and pressure in the liquid will determine the evolution of the system, which, in general, is quite different from the evolution we have when the coupling between the flow and deformation of the porous medium is absent, i.e., when the solid is rigid. This process (flow in non-deformable porous media) has been studied in several works. We refer, for instance, to [5] for the modeling and to [6], [7] for the analysis of the related mathematical problems.

Our study starts from the paper of Sommer and Mortensen [8] who analyzed the one-dimensional problem of the infiltration of a liquid in a deformable porous medium under the assumption that the driving pressure is high in comparison with the capillary pressures. They developed the simulation of the process and performed the experimental validation of the model. We focus, instead, on the mathematical modeling and on the analytical aspects of the problem. This, in fact, is a complicated free boundary problem characterized by the presence of two moving boundaries. As in [8] we do not consider the dynamic of the system in the short initial transient and assume that the porous medium is elastic. In fact, in the very first moment of contact between the liquid and the preform there is a transient period during which the liquid is strongly decelerated by the porous medium, which simultaneously is compressed to a volume fraction corresponding to the applied pressure. Accordingly, at time $t=0$, the infiltration front is at the entrance of the porous material which is compressed under the full applied pressure. Later, at $t>0$, the infiltration front passes, it engulfs a slice of the porous material which relaxes somewhat, following the unloading stress-strain curve. Therefore, in the one-dimensional case, we are in the presence of two free boundaries: one is given by the infiltration front and the other is given by the material surface of the preform which, relaxing, moves into the fluid.

In more detail, the paper develops as follows: after this introduction, the second section presents the general model based on the isothermal theory of deformable porous media. We recall briefly the three-dimensional infiltration model and the boundary conditions that have to be defined. In the third section we consider the one-dimensional problem getting to the formulation of the mathematical problem first in the Eulerian formalism and then in the Lagrangian formalism. The use of a set of material coordinates fixed on the solid (Lagrangian formalism) allows us, in fact, to simplify significantly the problem. 
Finally, the fourth section deals with the mathematical analysis of the problem obtained in the Lagrangian formalism. Here the existence of a solution is shown. To obtain this result we prove that the problems admit a self-similar solution.

\section{The mathematical model.}

2.1. General problem statement and physical assumptions. We obtain the general equations for the flow in deformable porous media from the theory of mixtures (see [9] or [10] for deeper expositions or [11] for a wide review on the subject).

The theory of mixtures is a theory based on balance laws and conservation principles which is well known in continuum mechanics. It can be applied to several scientific fields which have in common the fact that the system is constituted by a mixture of continua interacting at a microscopic scale.

In the isothermal case and in the absence of chemical reactions or phase change, the theory, following the Eulerian formalism, gives rise to the following system of independent equations:

$$
\begin{gathered}
\frac{\partial \phi_{s}}{\partial t}+\nabla \cdot\left(\phi_{s} \vec{v}_{s}\right)=0, \\
\frac{\partial \phi_{l}}{\partial t}+\nabla \cdot\left(\phi_{l} \vec{v}_{l}\right)=0, \\
\rho_{s} \phi_{s}\left(\frac{\partial \vec{v}_{s}}{\partial t}+\vec{v}_{s} \cdot \nabla \vec{v}_{s}\right)=\nabla \cdot \widetilde{\mathbf{T}}_{s}+\rho_{s} \phi_{s} \vec{g}+\vec{m}_{s}^{\sigma}, \\
\rho_{l} \phi_{l}\left(\frac{\partial \vec{v}_{l}}{\partial t}+\vec{v}_{l} \cdot \nabla \vec{v}_{l}\right)=\nabla \cdot \widetilde{\mathbf{T}}_{l}+\rho_{l} \phi_{l} \vec{g}+\vec{m}_{l}^{\sigma},
\end{gathered}
$$

where $s$ stands for the solid phase and $l$ for the liquid phase. Furthermore, referring to the $p$ constituent,

- $\rho_{p}$ is the "true" density, that is, the density of the material that is used as $p$ constituent of the mixture (e.g., $\rho_{\text {water }}=1000 \mathrm{Kg} / \mathrm{m}^{3}$ ) and it is considered constant (i.e., the materials are assumed incompressible);

- $\phi_{p}$ is the volume fraction, i.e., the volume occupied by the $p$ constituent over the unit volume. Therefore, assuming saturation,

$$
\phi_{s}+\phi_{l}=1
$$

- $\vec{v}_{p}$ is the velocity of the $p$ constituent;

- $\widetilde{\mathbf{T}}_{p}$ is the partial stress tensor, which describes the behaviour of the $p$ constituent when the other is co-present. The difference between the partial stress $\widetilde{\mathbf{T}}_{p}$ and the stress $\mathbf{T}_{p}$ of the $p$ constituent taken separately, usually named Reynolds stress, is well emphasized by a comparison with the model obtained using the ensemble average approach [11];

- $\vec{g}$ is the gravitational acceleration;

- $\vec{m}_{p}^{\sigma}$ is the momentum supply [12] (named also internal body force or interaction force) and is related to the local interactions between the $p$ constituent and the other constituent across the interface separating them. Since $\vec{m}_{p}^{\sigma}$ represents internal forces, we have that $\vec{m}_{s}^{\sigma}+\vec{m}_{l}^{\sigma}=0$. 
The unknowns of the system (1)-(4) are: $\phi_{s} ; \phi_{l} ; \vec{v}_{s} ; \vec{v}_{l}$. Condition (5) means, however, that they are not linearly independent. In particular, if we introduce the so-called composite velocity $\vec{v}_{c}$, defined as

$$
\vec{v}_{c}=\phi_{s} \vec{v}_{s}+\phi_{l} \vec{v}_{l}
$$

Eq. (5), adding (1) and (2), implies that

$$
\nabla \cdot \vec{v}_{c}=0
$$

which is a constraint that the solution $\left(\phi_{s} ; \phi_{l} ; \vec{v}_{s} ; \vec{v}_{l}\right)$ of the system (1)-(4) must satisfy. We can note also that in this way a divergence-free field is defined. In handling the model it is very useful to exploit this property, using (7) to get boundary conditions.

The presence of such a constraint implies the introduction of a Lagrange multiplier $\lambda$ which has to be determined. In this sense the system (1)-(4) is consistent: 8 independent equations for 8 independent unknowns $\lambda ; \phi_{l}$ (or $\phi_{s}$ ); $\vec{v}_{s} ; \vec{v}_{l}$.

Defining now

$$
\rho_{m}=\rho_{s} \phi_{s}+\rho_{l} \phi_{l}
$$

and

$$
\vec{v}_{m}=\frac{\rho_{s} \phi_{s} \vec{v}_{s}+\rho_{l} \phi_{l} \vec{v}_{l}}{\rho_{m}}
$$

respectively, as the mixture density and the mixture velocity (i.e., the velocity of the center of mass of the mixture) we obtain, from (1) and (2), the usual mass balance low for the mixture as a whole,

$$
\frac{\partial \rho_{m}}{\partial t}+\nabla \cdot\left(\rho_{m} \vec{v}_{m}\right)=0
$$

and, from (3), (4), and (10), the momentum equation for the mixture

$$
\rho_{m}\left(\frac{\partial \vec{v}_{m}}{\partial t}+\vec{v}_{m} \cdot \nabla \vec{v}_{m}\right)=\nabla \cdot \mathbf{T}_{m}+\rho_{m} \vec{g},
$$

where $\mathbf{T}_{m}$ represents the stress tensor of the mixture as a whole as it is linked to $\widetilde{\mathbf{T}}_{s}$ and $\widetilde{\mathbf{T}}_{l}$ by these relations:

$$
\begin{aligned}
& \mathbf{T}_{m}=\widetilde{\mathbf{T}}_{m}-\rho_{s} \phi_{s} \vec{u}_{s} \otimes \vec{u}_{s}-\rho_{l} \phi_{l} \vec{u}_{l} \otimes \vec{u}_{l}, \\
& \widetilde{\mathbf{T}}_{m}=\widetilde{\mathbf{T}}_{s}+\widetilde{\mathbf{T}}_{l},
\end{aligned}
$$

$\vec{u}_{s}=\vec{v}_{s}-\vec{v}_{m}$ and $\vec{u}_{l}=\vec{v}_{l}-\vec{v}_{m}$ being the so-called diffusive velocities. We can note that $\mathbf{T}_{m}$ can be determined by performing experiments on the mixture as a whole, while neither $\widetilde{\mathbf{T}}_{s}$ nor $\widetilde{\mathbf{T}}_{l}$ can be measured directly. Moreover, it is possible to show [13] that the Lagrange multiplier $\lambda$ is given by the isotropic part of $\mathbf{T}_{m}$, i.e., by $\operatorname{Tr}\left(\mathbf{T}_{m}\right)$, and therefore is physically interpreted as a hydrostatic pressure acting on the mixture. It is usually called pore pressure and denoted by $P$. Consequently we can write

$$
\mathbf{T}_{m}=-P \mathbf{I}+\mathbf{T}_{m}^{\prime}
$$

where $\mathbf{T}_{m}^{\prime}$ is called excess stress.

Obviously, the model must be closed giving $\mathbf{T}_{m}$, which, as mentioned, can be determined experimentally, and postulating constitutive relations for $\widetilde{\mathbf{T}}_{s}$ and $\vec{m}_{s}^{\sigma}$ (or $\widetilde{\mathbf{T}}_{l}$ 
and $\vec{m}_{l}^{\sigma}$ ). Ref. [11], for example, reports on some constitutive relations that have been assumed for some specific materials.

Anyway, even in the simple one-dimensional case, the mathematical problems that arise using the full model (1)-(4) are extremely difficult to handle. Therefore, it is customary to operate some simplifications based on certain physical assumptions. In practice the assumptions that are usually done are the following (see [9], for example):

A1. isotropic partial stress tensor for the liquid constitutent, i.e, $\widetilde{\mathbf{T}}_{l} \approx-\phi_{l} P \mathbf{I}$;

A2. negligible inertia of the constituents compared with the pressure gradient;

A3. besides a term $-P \nabla \phi_{p}$, the interaction force $\vec{m}_{p}^{\sigma}$ depends linearly on $\vec{v}_{s}-\vec{v}_{l}$ through an invertible tensor, which, in turn, depends on the deformation gradient $\mathbf{F}_{s}$ of the solid skeleton;

A4. gravitational effects can be neglected;

A5. surface tension and capillary effects can be neglected.

Under these assumptions, Eqs. (1) and (2) are not modified while in place of (3) and (4) we can write

$$
\begin{gathered}
\vec{v}_{l}-\vec{v}_{s}=-\frac{\mathbf{K}\left(\mathbf{F}_{s}\right)}{\mu \phi_{l}} \nabla P, \\
\nabla \cdot \mathbf{T}_{m}^{\prime}-\nabla P=0,
\end{gathered}
$$

where $\mu$ is the liquid viscosity and $\mathbf{K}$ is the permeability tensor depending on the deformation gradient $\mathbf{F}_{s}$ relative to the solid constitutent: $\left(\mathbf{F}_{s}\right)_{i \alpha}=\frac{\partial x_{i}}{\partial \xi_{\alpha}}, \vec{x} \equiv\left(x_{1} ; x_{2} ; x_{3}\right)$ being the actual coordinates and $\vec{\xi} \equiv\left(\xi_{1} ; \xi_{2} ; \xi_{3}\right)$ the coordinates in the reference configuration. Equation (15) is known as Darcy's law and Eq. (16) is noting more than the equilibrium of stresses.

In Sec. 3 this model will be specialized for the one-dimensional case and the mathematical problem that we get will be analyzed in the last section. It is important to remark again that Eqs. (15) and (16) can be considered a good approximation only if the physical assumptions, used for their deduction, are correct (see, for instance, [14] for a critical discussion of their validity). In particular, Eq. (15), i.e, Darcy's law, implies that the only interaction between solid and fluid is due to drag. This may not be correct unless the flow is very slow: i.e., the Reynolds number characterizing the flow is much less than 1. In summary, Darcy's law can be regarded as a consequence of the equation of motion for the fluid, together with specific assumptions about the nature of partial stress, interaction force, and effect of inertia. On the other hand, if Eq. (15) reduces the range of applicability of the model to those phenomena in which the above-listed assumptions are verified, it mitigates the problem of identifying the correct boundary conditions. In fact, as pointed out in [10], [15], and [16], Darcy's law, giving a link between the pressure gradient and the velocity of the fluid with respect to the solid, allows us to avoid the thorny problem of prescribing boundary conditions for traction boundary value problems.

2.2. Interface and boundary conditions. Since we want to study an infiltration problem we deal, in general, with a free boundary problem. We need, therefore, to specify the free boundaries evolution equations and the boundary conditions that we must impose on them. This will be coherently done while still working under the assumptions A1-A5. 
Let $\Omega$ be the domain occupied by the porous medium. In general $\Omega$ is divided into two time-varying sub-domains $\Omega^{d}$ and $\Omega^{w}$ corresponding, respectively, to the dry and to the wet part of $\Omega$. These two regions are separated by an interface denoted by $\sigma_{f}$.

The boundary $\partial \Omega$ presents, in general, also a second free boundary which is the contact interface between the pure liquid and the wet porous solid, and it is denoted by $\sigma_{e}$.

To find in a rigorous way the governing equations on $\sigma_{f}$ and $\sigma_{e}$ one has to proceed as specified in [17] or in Chapter 5 of [10]. One considers that the regions are separated by a transitional layer of thickness $\delta$ that has different properties of the mixture on its sides. For $\delta$ tending to zero the transitional layer shrinks to a surface and so one obtains the appropriate equations on the surface. Indeed, such equations are deduced by imposing, for each constitutent, the usual balance equations of the mass, momentum, momentum of momentum and energy. Anyway, this method, although rigorous, is quite involved and so we have preferred to proceed in a more heuristic way. Assuming that the thickness of the transitional layer between $\Omega^{w}$ and $\Omega^{d}$ is very small we have that $\sigma_{f}$ is a surface given by

$$
f_{f}(\vec{x}, t)=0
$$

The surface $\sigma_{e}$ is, instead, given by

$$
f_{e}(\vec{x}, t)=0 .
$$

Noting that $\sigma_{f}$ and $\sigma_{e}$ are material surfaces, respectively, for the liquid and for the solid, their evolution equations are simply given by

$$
\begin{aligned}
& \frac{\partial f_{f}}{\partial t}+\vec{v}_{l}\left(\sigma_{f}\right) \cdot \nabla f_{f}=0 \\
& \frac{\partial f_{e}}{\partial t}+\vec{v}_{s}\left(\sigma_{e}\right) \cdot \nabla f_{e}=0
\end{aligned}
$$

$\vec{v}_{l}\left(\sigma_{f}\right)$ and $\vec{v}_{s}\left(\sigma_{e}\right)$ being the liquid and solid velocity on $\sigma_{f}$ and $\sigma_{e}$.

Obviously (19) and (20) are kinematical constraints and so they do not supply any information on the conditions to impose to the physical quantities on the surfaces. Therefore, to obtain such kind of information (necessary to get a correct formulation of the mathematical problem) we impose the usual jump conditions on the mixture as a whole with inertia neglected:

$$
\begin{gathered}
\llbracket \rho_{m}\left(\vec{v}_{m}-\vec{v}(\sigma)\right) \cdot \vec{n}_{\sigma} \rrbracket=0, \\
\llbracket \mathbf{T}_{m} \vec{n}_{\sigma} \rrbracket=0,
\end{gathered}
$$

where

$$
\llbracket \cdot \rrbracket=(\cdot)^{+}-(\cdot)^{-}
$$

and

$\vec{v}(\sigma)$ and $\vec{n}_{\sigma}$ are the velocity and the normal of the surface $\sigma$.

We remark that Eqs. (21) and (22) can be derived from the general theory, assuming, as done, that $\sigma_{f}$ and $\sigma_{e}$ are plane material surfaces and neglecting all the surface properties. We refer to [10], Secs. 5.2 and 5.3, for a couple of examples in which the general theory, conveniently simplified, is used. 
2.2.1. Boundary conditions on $\sigma_{f}$. Since $\sigma_{f}$ is a material surface for the liquid, $\vec{v}_{l}$ is continuous across $\sigma_{f}$, and

$$
\llbracket \vec{v}_{l} \cdot \vec{n}_{f} \rrbracket=0
$$

where $\vec{n}_{f}$ is the outward normal of $\sigma_{f}$.

From (21) we have

$$
\llbracket \phi_{s}\left(\vec{v}_{s}-\vec{v}_{l}\left(\sigma_{f}\right)\right) \cdot \vec{n}_{f} \rrbracket=0 .
$$

From (22), following [18] and recalling (14) we obtain

$$
\begin{aligned}
\llbracket P \rrbracket & =0, \\
\llbracket \mathbf{T}_{m}^{\prime} \vec{n}_{f} \rrbracket & =0 .
\end{aligned}
$$

2.2.2. Boundary conditions on $\sigma_{e}$. The surface $\sigma_{e}$ is the interface between the pure liquid and the wet solid. If the superscript "+" denotes the quantities evaluated in the pure liquid region, then we have

$$
\begin{aligned}
\phi_{l}^{+} & =1, & \vec{v}_{l}^{+} & =\vec{u}_{\mathrm{in}}, \\
\mathbf{T}_{m}^{\prime+} & =0, & P^{+} & =P_{o},
\end{aligned}
$$

$\vec{u}_{\text {in }}$ being the velocity of the liquid penetrating into the preform and $P_{o}$ the driving liquid pressure. As specified, $\sigma_{e}$ is a material surface for the solid and so its velocity is the solid velocity evaluated on $\sigma_{e}$ itself. Taking account of that, (21) implies that

$$
\vec{u}_{\text {in }} \cdot \vec{n}_{e}=\left(\phi_{l}^{-} \vec{v}_{l}^{-}+\phi_{s}^{-} \vec{v}_{s}^{-}\right) \cdot \vec{n}_{e}
$$

where $\vec{n}_{e}$ is the outward normal of $\sigma_{e}$ and the superscript "-" denotes the quantities evaluated in the infiltrated region.

From (22) we get two conditions analogous to (25) and (26), which can be explicitly written as

$$
\begin{aligned}
P^{-} & =P_{o}, \\
\mathbf{T}_{m}^{\prime} \vec{n}_{e} & =0 .
\end{aligned}
$$

Note that (29) expresses the absence, on $\sigma_{e}$, of normal stress in the solid.

As in describing the behaviour of two interfacing continua one should impose the noslip condition, in solving problems involving flows over porous media one has to impose a condition that gives the relation between the tangential components of the velocities. Anyway, we remark that, in the context of the theory of mixture, the issue of the appropriate boundary conditions is far from being settled. For example, in Sec. 4.1 of [10], two methods for obtaining boundary conditions are discussed: the first one appeals to thermodynamics and holds when the mixture on the boundary is saturated; the second one is based on the splitting of the traction. Other examples of boundary conditions can be found in [19] and [20]. Reference [19], for instance, investigates the boundary conditions for a flux at a permeable wall. However, we do not need to examine these methods since we are going to deal with one-dimensional problems and so the conditions that have been stated are sufficient to get a correct formulation of the mathematical problem. 
3. The one-dimensional mathematical problem. In this section we consider the one-dimensional case. At first, we present the problem that is obtained using the Eulerian formalism; then we will get to a simplified version of it by using a set of Lagrangian coordinates fixed on the solid skeleton.

3.1. The Eulerian formulation. Let us consider a one-dimensional porous medium of length $L$. Let $x$ be the coordinate along the medium. We assume that the porous medium is elastic, initially homogeneous, isotropic, and that the flow and the strain take place only along the $x$-axis. Let $\phi_{r}$ be its solid volume fraction in the non-stressed configuration. At $t=0$ a constant, the known pressure $P_{o}$ is applied on one end of the solid, which immediately compresses, reducing its length to $L_{c}$ and acquiring a solid volume fraction $\phi_{s}=\phi_{c}$ yielding a stress that balances the applied pressure. As in [8], we study the evolution of the system from this instant and we place the origin $x=0$ at the position to which the preform entrance is compressed.

Denoting by $\tau$ the $(x, x)$-component of $\mathbf{T}_{m}^{\prime}$, counted as positive in compression (in practice $\left.\left(\mathbf{T}_{m}^{\prime}\right)_{x x}=-\tau\right)$ and by $K$ the $(x, x)$-component of $\mathbf{K}$ and defining, in order to have lighter notation, $\phi_{s}$ as $\phi$, Eqs. (1), (2), (15), and (16) can be rewritten in the following way:

$$
\begin{aligned}
& \frac{\partial \phi}{\partial t}+\frac{\partial}{\partial x}\left(\phi v_{s}\right)=0, \\
& \frac{\partial \phi}{\partial t}-\frac{\partial}{\partial x}\left(v_{l}(1-\phi)\right)=0 \\
& v_{l}-v_{s}=-\frac{K}{\mu(1-\phi)} \frac{\partial P}{\partial x} \\
& \frac{\partial P}{\partial x}=-\frac{\partial \tau}{\partial x}
\end{aligned}
$$

To close the system (30)-(33) we need constitutive equations for $\tau$ and $K$. The elasticity assumption means that the stress tensor $\tau$ is a function of the deformation gradient of the solid which, in one dimension, is given by

$$
\left(\mathbf{F}_{s}\right)_{x x}=\frac{\partial x}{\partial \xi}=\frac{\phi_{c}}{\phi}
$$

where the reference configuration is the initial one in which the solid is compressed but still not infiltrated. For instance, in [8], [21], [22], and [23], stress-strain curves for some porous materials like polyurethane sponge are reported. We assume, according to these experimental results, that $\tau(\phi)$ is $C^{1}$ and that

$$
\Sigma=\frac{d \tau(\phi)}{d \phi}>0
$$

Therefore, under such hypotheses, we have that

$$
\phi_{c}=\tau^{-1}\left(P_{o}-P_{\text {atm }}\right)
$$

where $\tau^{-1}$ is the inverse function of $\tau, P_{\text {atm }}$ is the atmospheric pressure and $P_{o}$, as mentioned, the applied pressure.

For the permeability $K$, from assumption A3 and from (34), one obtains

$$
K=K(\phi) \text {. }
$$


In [8], for instance, the behaviour of $K$ versus $\phi$ for a polyurethane sponge is reported.

Once specified, the constitutive relations for $\tau$ and $K$ in the equations (30)-(33) have to be solved in the spatial domain $\Omega$ occupied by the porous solid. Since we are concerned with an infiltration problem, $\Omega$, as mentioned in Sec. 2.2 , is divided into two time-varying sub-domains:

$$
\begin{aligned}
\Omega^{w} & =\left\{x_{e}(t)<x<x_{f}(t)\right\}, \\
\Omega^{d} & =\left\{x_{f}(t)<x<L_{c}\right\},
\end{aligned}
$$

corresponding, respectively, to the wet and the dry parts of the porous solid. The boundary $x_{f}(t)$ is, in fact, the interface between the wet and the dry part and $x_{e}(t)$ is the entrance of the preform. In $\Omega^{w}$ the fluid is the infiltrating liquid; in $\Omega^{d}$ the fluid is the air.

The whole infiltration problem consists, therefore, in solving in $\Omega^{w}$ and $\Omega^{d}$ two systems of equations given by (30)-(33) (coupled by suitable conditions specified on $x_{f}$ ) and the evolution equations for the free boundaries $x_{e}(t)$ and $x_{f}(t)$.

The remaining part of this section is devoted to specifying in detail the equations in both sub-domains and to determining the evolution equations for the interfaces $x_{f}$ and $x_{e}$.

Dry region $\Omega^{d}$. If we assume, in addition to the hypotheses $\mathrm{A} 1-\mathrm{A} 5$, that in $\Omega^{d}$ :

B1. the solid stress tensor $\tau$ is equal to that one in the wet region (i.e., same constitutive equations);

B2. the interaction between the air and the solid can be neglected so that there is no pressure drop in the air $P=P_{\text {atm }}$;

then we have the following equations:

$$
\begin{aligned}
\frac{\partial \phi}{\partial t}+\frac{\partial}{\partial x}\left(\phi v_{s}\right) & =0 \\
\frac{\partial \phi}{\partial t}+\frac{\partial}{\partial x}\left(v_{\text {air }}(1-\phi)\right) & =0 \\
\frac{\partial \tau(\phi)}{\partial x} & =0
\end{aligned}
$$

where the unknowns are: $\phi, v_{s}$, and $v_{\text {air }}$, that is the air velocity.

It is worth noting that system (40)-(42) can be solved analytically. From (42) it follows, in fact, that

$$
\phi(x, t)=\phi^{d}(t) \quad \forall x \in \Omega^{d} .
$$

In particular, because of B1, (26) implies the continuity of $\phi$ across $\sigma_{f}$,

$$
\phi\left(x_{f}(t), t\right)=\phi^{d}(t) .
$$

In fact, integrating (33) from $x_{e}$ to $x_{f}$, we have

$$
\tau\left(\phi\left(x_{e}, t\right)\right)-\tau\left(\phi\left(x_{f}, t\right)\right)=-\left(P_{o}-P_{\mathrm{atm}}\right),
$$

which, because of the absence of stress on $x_{e}$ (i.e., $\tau\left(\phi\left(x_{e}, t\right)\right)=0$, see $\left.(29)\right)$ involves

$$
\phi\left(x_{f}, t\right)=\tau^{-1}\left(P_{o}-P_{\text {atm }}\right) .
$$


So, from (36), (43), and (44), we have determined $\phi$ in $\Omega^{d}$ :

$$
\phi(x, t)=\phi^{d}(t)=\phi_{c} \quad \forall x \in \Omega^{d} ; t>0,
$$

with $\phi_{c}$ constant in time, $P_{o}$ being constant. Note also that (45) allows us to determine easily the boundary condition on $x_{f}$. In fact, (43) entails

$$
\phi\left(x_{f}(t), t\right)=\phi_{c}
$$

which in turn implies, because of (23) and (24), the continuity of the solid velocity $v_{s}$ across $x_{f}$.

Now the air velocity in $\Omega^{d}$ can readily be found. In fact, Eqs. (41) and (23) imply that

$$
v_{\text {air }}(x, t)=\dot{x}_{f}(t) \quad \forall x \in \Omega^{d} ; t>0,
$$

$\dot{x}_{f}$ being the velocity of the liquid on $x_{f}$, which, as stated, is a material surface for the liquid itself.

Finally, the third unknown, i.e., $v_{s}$, can be determined using (40), (45) and the fact that the end-side of the dry solid is fixed. We have

$$
v_{s}(x, t)=0 \quad \forall x \in \Omega^{d} ; t>0,
$$

which in turn, because of continuity (mentioned above) of $v_{s}$ across $x_{f}$, implies that

$$
\lim _{\substack{x \rightarrow x_{f} \\ x \in \Omega^{w}}} v_{s}(x, t)=0 .
$$

Wet region $\Omega^{w}$. In the wet region $\Omega^{w}$ the problem cannot be solved analytically. Anyway, the system (30)-(33) can be reduced to one equation in the unknown $\phi$. Recalling, in fact, that the composite velocity $v_{c}$, defined in (6), is a divergence-free field, using (30), (32), and (35), we reduce (30)-(33) to the following parabolic equation:

$$
\frac{\partial \phi}{\partial t}=\frac{\partial}{\partial x}\left[\phi \frac{K(\phi)}{\mu} \Sigma(\phi) \frac{\partial \phi}{\partial x}\right]-v_{c} \frac{\partial \phi}{\partial x},
$$

to which one has to add the boundary conditions on $x_{e}(t), x_{f}(t)$ and the expression of $v_{c}$ in terms of $\phi$.

The boundary condition on $x_{e}$ is deduced from (29), which implies the absence of stress in the solid on $x_{e}$. Consequently, assuming, as mentioned above, that the non-stressed configuration of the solid corresponds to $\phi=\phi_{r}$, we have

$$
\phi\left(x_{e}(t), t\right)=\phi_{r}
$$

The boundary condition on $x_{f}$ is given by (46).

The composite velocity $v_{c}$ can be linked to $u_{\text {in }}$ and $\dot{x}_{f}$. In fact, because of (7), we have $v_{c}=v_{c}(t)$ and from $(24),(27)$ we obtain

$$
v_{c}=u_{\mathrm{in}}(t)=\left(1-\phi_{c}\right) \dot{x}_{f}(t)
$$

where, as stated below $(47), v_{l}\left(x_{f}, t\right)=\dot{x}_{f}$. 
Free boundaries equations. From (19) and (20), it is possible to deduce the evolution equations for the free boundaries $x_{e}, x_{f}$ in terms of $\phi$. Let us first focus on $x_{f}$. Using Darcy's law, (32), and (33), we have the following Stefan-type condition:

$$
\dot{x}_{f}=\left.\frac{K\left(\phi_{c}\right)}{\mu\left(1-\phi_{c}\right)} \Sigma\left(\phi_{c}\right) \frac{\partial \phi}{\partial x}\right|_{x_{f}} .
$$

Using again Darcy's law, (27), and (51), we get the following evolution equation for $x_{e}$ :

$$
\dot{x}_{e}=\left.\frac{K\left(\phi_{c}\right)}{\mu} \Sigma\left(\phi_{c}\right) \frac{\partial \phi}{\partial x}\right|_{x_{f}}-\left.\frac{K\left(\phi_{r}\right)}{\mu} \Sigma\left(\phi_{r}\right) \frac{\partial \phi}{\partial x}\right|_{x_{e}} .
$$

In conclusion, the dynamic of the whole system is governed, in the Eulerian formalism, by a nonlinear free boundary problem defined in $\Omega^{w}$. In particular, the equations that define the problem are: (49) with $v_{c}$ given by (51), (46), (50), (52), and (53) with the initial conditions $x_{e}(0)=x_{f}(0)=0$.

3.2. The Lagrangian formulation. The problem stated above is highly nonlinear and characterized by the presence of two free boundaries, which give rise to several mathematical difficulties. Therefore, in order to reduce them, we consider a set of material coordinates fixed on the solid. In this reference, one boundary ( $x_{e}$ for precision) is fixed and so we get a nonlinear one-phase Stefan problem. Let $\xi$ be the independent variable that labels the solid particles and $x=x(\xi, t)$ its position at time $t$. As is well known in continuum mechanics, $\frac{\partial x(\xi, t)}{\partial \xi} \neq 0 \forall \xi \in\left[0, L_{c}\right]$ and $\forall t \geq 0$. As reference configuration we consider the one at time $t=0$, when the solid is completely compressed but still not infiltrated. We have, therefore: $x(\xi, 0)=\xi, 0 \leq \xi \leq L_{c}$ and $\phi(\xi, 0)=\phi_{c}$.

Instead of $\phi$, in the Lagrangian formulation, it is useful to consider, as dependent variable, the so-called void ratio defined as

$$
e=\frac{1-\phi}{\phi}
$$

In the reference configuration, $e(\xi, 0)=e_{c}=\left(1-\phi_{c}\right) / \phi_{c}$. It is also useful, since in the following we will work always with $e$, to consider $\tau$ and $K$ as functions of $e$, i.e., $K=K(e)$ and $\tau=\tau(e)$.

We introduce also the function $\widetilde{\Sigma}(e)$ defined as

$$
\widetilde{\Sigma}(e)=\frac{d \tau(e)}{d e},
$$

which, because of (35) and (54), is negative.

Obviously, using Lagrangian coordinates fixed on the solid, the wet domain becomes $\Omega^{w}=\left\{0 \leq \xi \leq \xi_{f}(t)\right\}$ where $\xi_{f}(t)$ denotes the solid particle just wet by the infiltration front, i.e., it locates the free boundary between the wet and the dry domain. The "old" free boundary $x_{e}$ has disappeared, being now given by $\xi=0$. This is the biggest advantage in using Lagrangian coordinates: one boundary is fixed.

To deduce the dynamics in the Lagrangian formalism we consider Eqs. (31)-(33) and (7) (instead of the whole system (30)-(33)) and we transform them into equations involving Lagrangian quantities. Recalling (34), the definition (54), and the rule of 
derivative of composite functions,

$$
\frac{\partial(\cdot)}{\partial x}=\frac{\partial \xi}{\partial x} \frac{\partial(\cdot)}{\partial \xi}=\frac{e_{c}+1}{e+1} \frac{\partial(\cdot)}{\partial \xi},
$$

Eqs. (7), (32), and (33) become, respectively,

$$
\begin{aligned}
& \frac{\partial v_{c}}{\partial \xi}=0 \\
& v_{l}-v_{s}=-\frac{e_{c}+1}{e} \frac{K(e)}{\mu} \frac{\partial P}{\partial \xi} \\
& \frac{\partial P}{\partial \xi}=-\frac{\partial \tau(e)}{\partial \xi}
\end{aligned}
$$

More attention has to be posed in transforming (31) into Lagrangian coordinates. To determine the mass balance equation for the liquid phase in the Lagrangian formalism, one has to consider a volume fixed on the solid skeleton and write the liquid mass balance with respect to this volume. The general three-dimensional equation is

$$
\left(\frac{d e}{d t}\right)_{s}+\nabla_{\vec{\xi}} \cdot\left[e \mathbf{F}_{s}^{-1}\left(\vec{v}_{l}-\vec{v}_{s}\right)\right]=0,
$$

where $\left(\frac{d}{d t}\right)_{s}$ is the material derivative following the solid, $\nabla_{\vec{\xi}}$. is the divergence operator with respect to $\vec{\xi}$, and $\mathbf{F}_{s}^{-1}$ is the inverse of the solid deformation gradient $\mathbf{F}_{s}$. So, in one dimension, because of (34), we get

$$
\frac{\partial e}{\partial t}+\left(1+e_{c}\right) \frac{\partial}{\partial \xi}\left[\frac{e}{1+e}\left(v_{l}-v_{s}\right)\right]=0,
$$

which has to be added to the system (56)-(58).

Substituting now (57) into (59) and taking account of (58) and (55), we obtain

$$
\frac{\partial e}{\partial t}+\frac{\left(1+e_{c}\right)^{2}}{\mu} \frac{\partial}{\partial \xi}\left[\frac{K(e)}{(1+e)} \tilde{\Sigma}(e) \frac{\partial e}{\partial \xi}\right]=0,
$$

which is the governing equation in the Lagrangian formalism.

The boundary conditions can easily be deduced from (46) and (50), recalling that $x_{e}(t)$ corresponds to $\xi=0$ and $x_{f}(t)$ to $\xi_{f}(t)$. So we have

$$
\begin{aligned}
e(0, t) & =e_{r}, \\
e\left(\xi_{f}(t), t\right) & =e_{c},
\end{aligned}
$$

where $e_{r}=\left(1-\phi_{r}\right) / \phi_{r}$ and $e_{c}=\left(1-\phi_{c}\right) / \phi_{c}$.

Finally, we have to write explicitly the evolution equation for the free boundary $\xi_{f}(t)$. This can easily be done by observing that $\dot{\xi}_{f}(t)$ is the interface velocity with respect to the solid and therefore

$$
\dot{\xi}_{f}(t)=v_{l}\left(x_{f}(t), t\right)-v_{s}\left(x_{f}(t), t\right),
$$

which, using (55), (57), (58), and (62), rewrites

$$
\dot{\xi}_{f}(t)=\left.\frac{K\left(e_{c}\right)}{\mu} \frac{e_{c}+1}{e_{c}} \widetilde{\Sigma}\left(e_{c}\right) \frac{\partial e}{\partial \xi}\right|_{\xi_{f}(t)} .
$$


In conclusion, the dynamics, in the Lagrangian formalism, is governed by the free boundary problem: (60)-(63) whose initial condition is $\xi_{f}(0)=0$.

We can compare this problem with the previous one obtained in the Eulerian formalism and notice the great simplification that the use of material coordinates fixed on the solid has involved. The problem, in Lagrangian coordinates, although nonlinear, is, in fact, a classical Stefan problem and so there are several analytical techniques that can be applied. In the next section, we develop the analysis of such a problem.

4. Similarity solutions. For the sake of simplicity we define

$$
F(e)=-\frac{\left(e_{c}+1\right)^{2}}{\mu(e+1)} K(e) \widetilde{\Sigma}(e),
$$

which, according to the assumptions made in the previous section, turns out to be strictly positive. Rewriting the free boundary problem (60)-(63) as

$$
\begin{array}{ll}
\frac{\partial e}{\partial t}=\frac{\partial}{\partial \xi}\left[F(e) \frac{\partial e}{\partial \xi}\right], & 0<\xi<\xi_{f}(t), t>0, \\
e(0, t)=e_{r}, & t>0, \\
e\left(\xi_{f}(t), t\right)=e_{c}, & t>0, \\
\dot{\xi}_{f}=-\left.\frac{F\left(e_{c}\right)}{e_{c}} \frac{\partial e}{\partial \xi}\right|_{\xi_{f}(t)}, & t>0, \\
\xi_{f}(0)=0, &
\end{array}
$$

we realize that we are dealing with a type of one-phase Stefan problem. In fact, we can think of $e$ as a temperature, $e_{c}$ the melting point, and $F(e)$ a given non-constant thermal conductivity. We recall that boundary values satisfy: $0<e_{c}<e_{r}$. Therefore we can refer to all the classical literature concerning this problem (see, for instance, [24] or [25]).

In this section we are interested in looking for self-similar solutions of the problem. We introduce the Boltzmann transformation

$$
\chi=\frac{\xi}{\xi_{f}(t)},
$$

and we assume that there exists a real function $u$ such that the solution of $(64)-(68)$ could be written as

$$
e(\xi, t)=u(\chi), \quad 0<\chi<1 .
$$

By inserting this relation in (67), we have

$$
\xi_{f} \dot{\xi}_{f}=-\frac{F\left(e_{c}\right)}{e_{c}} u^{\prime}(1)
$$

where the right-hand side is a constant, though unknown yet. Then we can easily integrate the equation for the free boundary, obtaining the explicit formula:

$$
\xi_{f}(t)=\left(-\frac{2 F\left(e_{c}\right) u^{\prime}(1)}{e_{c}} t\right)^{1 / 2} .
$$


In order to get $u^{\prime}(1)$ we have to solve the equation for $u$ obtained by substituting $u$ instead of $e$ in (64) according to (70). What we get is the following limit problem:

$$
\begin{aligned}
& \frac{F\left(e_{c}\right)}{e_{c}} u^{\prime}(1) \chi u^{\prime}-\left(F(u) u^{\prime}\right)^{\prime}=0, \quad 0<\chi<1, \\
& u(0)=e_{r}, \\
& u(1)=e_{c} .
\end{aligned}
$$

A peculiarity of the differential equation is that it contains $u^{\prime}(1)$, which is the value of the derivative of the solution at the right end. To get around this aspect, we study the family of problems obtained by replacing $u^{\prime}(1)$ with the parameter $\alpha$. Then we could consider the set $\left\{u_{\alpha}\right\}$ of solutions of the standard elliptic problems on the unit interval and, among them, look for those that satisfy $u_{\alpha}^{\prime}(1)=\alpha$.

Unfortunately, this method requires the study of limit problems and we prefer to deal with the more familiar Cauchy's versions. Therefore, following the same scheme, we consider the following family of ordinary problems depending on $\alpha$ :

$$
\begin{aligned}
& \frac{F\left(e_{c}\right) \alpha}{e_{c}} \chi u^{\prime}-\left(F(u) u^{\prime}\right)^{\prime}=0, \quad 0<\chi<1, \\
& u(1)=e_{c}, \\
& u^{\prime}(1)=\alpha,
\end{aligned}
$$

and we determine $\alpha$ by imposing $u(0)=e_{r}$.

By integrating the differential equation between $\chi$ and 1 , we obtain finally the equivalent problem:

$$
\begin{aligned}
& u^{\prime}=\frac{\alpha F\left(e_{c}\right)}{e_{c} F(u)}\left(\chi u+\int_{\chi}^{1} u(s) d s\right), \\
& u(1)=e_{c} .
\end{aligned}
$$

Now we prove the following

TheOREm 4.1. Let $F$ be a positive Lipschitz function. Then problem (64)-(68) has a unique self-similar solution.

Proof. According to what we have already said, the problem is equivalent to that of finding a unique $\alpha \leq 0$ such that the solution $u_{\alpha}$ of (79) satisfies $u_{\alpha}(0)=e_{r}$.

It is clear that the thesis is a straightforward consequence of the following steps:

a) $u_{0} \equiv e_{c}$;

b) If $\alpha<0$ then $u_{\alpha}$ is strictly decreasing in [0,1];

c) if $\alpha<\bar{\alpha}$ then $u_{\alpha}(\chi)>u_{\bar{\alpha}}(\chi), 0 \leq \chi<1$;

d) the application $\alpha \mapsto u_{\alpha}(0)$ is continuous;

e) $u_{\alpha}(0) \rightarrow \infty$ when $\alpha \rightarrow-\infty$.

By putting all these ingredients together, we have that the application $\alpha \mapsto u_{\alpha}(0)$ is continuous, monotone, and unbounded when $\alpha \in(-\infty, 0]$. Therefore, there exists a unique value of $\alpha$ such that $u_{\alpha}(0)=e_{r}$.

Let us now go through each of the steps: a) is trivial. 
For any fixed $\alpha<0$, we prove first that $u_{\alpha}(\chi) \geq e_{c}$. We have $u^{\prime}(1)<0$, which means that at least in a left neighbour of $1, u_{\alpha}$ is strictly greater than $e_{c}$. We suppose by contradiction that there exists $0<\bar{\chi}<1$ such that $u_{\alpha}(\bar{\chi})=e_{c}$ and $u_{\alpha}(\chi)>e_{c}$ for $\bar{\chi}<\chi<1$. By (79) we would have $u_{\alpha}^{\prime}(\bar{\chi}) \leq \alpha<0$, which is absurd. Then $u_{\alpha}(\chi)>0$ for $0 \leq \chi<1$ and from $(79) u_{\alpha}^{\prime}(\chi) \leq \alpha$, which proves b).

Now, consider $\alpha<\bar{\alpha} \leq 0$ and let $w(\chi)=u_{\alpha}(\chi)-u_{\bar{\alpha}}(\chi)$; we have $w(1)=0$ and $w^{\prime}(1)=\alpha-\bar{\alpha}<0$. Again, $w$ is positive in a left neighbourhood of 1. Let us assume by contradiction that there exists $0<\bar{\chi}<1$ such that $w(\bar{\chi})=0$ and $w(\chi)>0$ for $\bar{\chi}<\chi<1$. We would have, by subtracting (79) relative to $\bar{\alpha}$ by that relative to $\alpha$,

$$
w^{\prime}(\bar{\chi})=\frac{F\left(e_{c}\right)}{e_{c} F(u(\bar{\chi}))}\left[(\alpha-\bar{\alpha})\left(\bar{\chi} u(\bar{\chi})+\int_{\bar{\chi}}^{1} u(s) d s\right)+\bar{\alpha} \int_{\bar{\chi}}^{1} w(s) d s\right]<0,
$$

that each term in square brackets is negative.

Again we end up with a contradiction that proves c).

Point d) is a consequence of the continuous dependence theorem of the solutions of ordinary differential equations and, finally, point e) follows from the previous relation $u_{\alpha}^{\prime}(\chi) \leq \alpha$, which entails $u_{\alpha}(0) \geq e_{c}-\alpha$.

Let us give some remarks concerning the characteristics of the solution $u$ of (73). We already know from point b) that $e_{c}<u(\chi)<e_{r}$ in $(0,1)$; by the way, since $F$ is strictly positive, this is also an immediate consequence of the maximum principle for elliptic problems. By considering the function $v(\chi)=u(\chi)-e_{r}+\chi\left(e_{r}-e_{c}\right)$ and applying again the same principle, we have that $v(\chi)<0$ in $(0,1)$. This means that our solution $u$, besides being monotone decreasing, lies beneath the straight line connecting the two extrema.

Now we want to go back to the original coordinates $(x, t)$ and obtain the formula for the motion of the two boundaries $x_{e}$ and $x_{f}$.

First of all, we recall that for any $t$ we have that $x(\xi, t)=\xi$ when $\xi_{f}(t) \leq \xi \leq 1$ and then we have immediately

$$
x_{f}(t)=\xi_{f}(t)=\left(-\frac{2 F\left(e_{c}\right) u^{\prime}(1)}{e_{c}} t\right)^{1 / 2}
$$

where now $u$ is the solution of (73).

In order to get the expression for the other boundary, we integrate (34) between 0 and $\xi_{f}$ and, by using (54), we get

$$
x_{f}(t)-x_{e}(t)=\phi_{c} \int_{0}^{\xi_{f}(t)}(e(\xi, t)+1) d \xi=x_{f}(t) \phi_{c}\left(1+\int_{0}^{1} u(\chi) d \chi\right)
$$

and then

$$
x_{e}(t)=-x_{f}(t)\left(\phi_{c}+\phi_{c} \int_{0}^{1} u(\chi) d \chi-1\right) .
$$

We see that $x_{e}(t)$ is simply proportional to $x_{f}(t)$, although it moves in the opposite direction, as it must be. In fact, we have that point b) of the previous proof entails

$$
\int_{0}^{1} u(\chi) d \chi>e_{c}
$$


and then, by using (54),

$$
\phi_{c}+\phi_{c} \int_{0}^{1} u(\chi) d \chi-1>\phi_{c}+\phi_{c} e_{c}-1=0 .
$$

5. Conclusions. In this paper we have analyzed a model which can be applied to those industrial processes involving flow through deformable porous materials and, in particular, to some processes (RTM, SRIM, or SC) used for manufacturing composite materials.

We have analyzed the coupled flow/deformation problem considering a set of material coordinates fixed on the solid. This technique has reduced the mathematical problem to a nonlinear one-phase Stefan problem, which has been studied showing the existence of a self-similar solution.

Acknowledgments. We gratefully acknowledge Prof. Luigi Preziosi, Prof. Nicola Bellomo (Politecnico di Torino), and Prof. Antonio Fasano (Università degli Studi di Firenze) for their continued assistance. The work was partially supported by the Italian Ministry for the University and Scientific Research (M.U.R.S.T.) and by the Italian National Research Council (C.N.R.) as a special project on "Dynamics of Multicomponent Systems".

\section{REFERENCES}

[1] R. K. Everett and J. R. Arsenault, Treatise on Material Science and Technology, Academic Press, 1991

[2] A. I. Isayev, Injection and Compression Molding Fundamentals, Marcel Dekker, 1987

[3] D. Purslow and R. Child, Autoclave molding of carbon-fiber reinforcing epoxies, Composites 17, 127-136 (1986)

[4] R. K. Upadhyay and E. W. Liang, Consolidation of advanced composites having volatile generation, Polymer Compos. 12, 96-108 (1995)

[5] A. E. Scheiddeger, Hydrodynamics in porous media, in Handbuck der Phisik, S. Flügge, C. Truesdell, eds., Vol. III/2, Springer-Verlag, 1963

[6] A. Passerini, Steady flows of a viscous incompressible fluid in a porous half-space, Math. Models and Meths. Appl. Sci. 4, 705-732 (1994)

[7] A. Lyaghfouri, The inhomogeneous dam problem with linear Darcy's law and Dirichlet boundary conditions, Math. Models and Meths. Appl. Sci. 6, 1051-1077 (1996)

[8] J. L. Sommer and A. Mortensen, Forced unidirectional infiltration of deformable porous media, J. Fluid. Mech. 311, 193-217 (1996)

[9] R. M. Bowen, The theory of mixtures, in Continuum Physics, A. C. Eringen, ed., Vol. 3, Academic Press, 1976

[10] K. R. Rajagopal and L. Tao, Mechanics of Mixtures, World Scientific, 1995

[11] L. Preziosi, The theory of deformable porous media and its application to composite material manufacturing, Surv. Math. Ind. 6, 167-214 (1996)

[12] C. A. Truesdell and R. A. Toupin, The classical field theory, in Handbuck der Phisik, S. Flügge, C. Truesdell, eds., Vol. III/1, Springer-Verlag, 1960

[13] R. M. Bowen, Incompressible porous media models by use of the theory of mixtures, Internat. J. Engrg. Sci. 18, 1129-1148 (1980)

[14] D. Munaf, S. Wineman, K. R. Rajagopal, and D. W. Lee, A boundary value problem in ground water motion analysis-comparison of predictions based on Darcy's law and the continuum theory of mixtures, Math. Models and Meths. Appl. Sci. 3, 231-248 (1993)

[15] K. R. Rajagopal, A. S. Wineman, and M. Gandhi, On boundary conditions for a certain class of problems in mixture theory, Internat. J. Engrg. Sci. 24, 1453-1463 (1986) 
[16] D. E. Kenyon, The theory of incompressible solid-fluid mixture, Arch. Rational Mech. Analysis 62, 131-174 (1976)

[17] L. Tao, K. R. Rajagopal, and A. S. Wineman, Unsteady diffusion of fluids through solids undergoing large deformations, Math. Models and Meths. Appl. Sci. 1, 311-346 (1991)

[18] I. S. Liu, On chemical potential and incompressible porous media, J. Mech. 19, 327-342 (1980)

[19] G. S. Beavers and D. D. Joseph, Boundary conditions at a naturally permeable wall, J. Fluid Mech. 30, 197-207 (1967)

[20] J. S. Hou, M. H. Holmes, W. M. Lai, and V. C. Mow, Boundary conditions at the cartilage-synovial fluid interface for joint lubrication and theoretical verifications, J. Biomech. Eng. 111, 78-87 (1989)

[21] Y. Lanir, S. Sauob, and P. Maretsky, Nonlinear finite deformation response of open cell polyurethane sponge to fluid filtration, Trans. ASME E: J. Appl. Mech. 57, 449-454 (1990)

[22] K. H. Parker, R. V. Mehta, and C. G. Caro, Steady flow in porous, elastically deformable materials, Trans. ASME E: J. Appl. Mech. 54, 794-800 (1987)

[23] Y. R. Kim, S. P. McCarthy, and J. P. Fanucci, Compressibility and relaxation of fiber reinforcements during composites processing, Polymer Compos. 12, 13-19 (1991)

[24] A. Fasano and M. Primicerio, Free boundary problems for nonlinear parabolic equations with nonlinear free boundary conditions, J. Math. Anal. Appl. 72, 247-273 (1979)

[25] A. M. Meirmanov, The Stefan Problem, De Gruyter, 1992 